\title{
Metastatic Angiosarcoma: A Rare Malignancy
}

\author{
Ramashish Kumar, Ashok K. Chauhan, Blessy K. Rajan and Diptajit Paul
}

\section{ABSTRACT}

\begin{abstract}
Angiosarcoma is an infrequent vascular malignancy with grave prognosis due to distant metastasis at initial presentation. The predominant metastatic sites of angiosarcoma are lung; and it is particularly important and should be approached with multimodality treatment because pulmonary metastasis of angiosarcoma frequently induces severe complications. Here, we reported a case of angiosarcoma in an old Indian male presented with cough and hemoptysis. 18-FDG PET-CT scan revealed multiple organ involvement by tumor, and excisional biopsy from lung and pleural nodule revealed malignant tumor composed of spindle shaped epithelioid cell. Strong positivity for D2-40, CD 31 \& CD 34 favoured the diagnosis of angiosarcoma. Patient was planned for intravenous chemotherapy with paclitaxel along with zoledronic acid. After 3-cycles of chemotherapy, re-evaluation with PET-CT revealed good response. In view of stable disease, it was planned to continue the same regimen for three more cycles. However, after receiving two more cycles of chemotherapy, patient's condition deteriorated rapidly and died within a month. The present case showed, paclitaxel has a good impact on metastatic angiosarcoma to diminish lung nodules. However, more effective treatment regimen should be explored to effectively treat metastatic angiosarcoma and increase survival.
\end{abstract}

Keywords: Angiosarcoma, lung metastasis, paclitaxel, zoledronic acid.
Published Online: October 25, 2021

ISSN: $2736-5476$

DOI: $10.24018 /$ ejclinicmed.2021.2.5.127

\section{R. Kumar}

Department of Radiation Oncology, Pt B D Sharma University of Health Sciences, Rohtak, Haryana, India.

(e-mail: ramashishkumar04@gmail.com) A. K. Chauhan

Department of Radiation Oncology, Pt B D Sharma University of Health Sciences, Rohtak, Haryana, India.

(e-mail: drchauhanashok@ yahoo.co.in)

B. K. Rajan

Department of Radiation Oncology, Pt B D

Sharma University of Health Sciences,

Rohtak, Haryana, India.

(e-mail: stephanie12399@yahoo.com)

D. Paul*

Department of Radiation Oncology, Pt B D Sharma University of Health Sciences, Rohtak, Haryana, India.

(e-mail: diptajitpaul.91@gmail.com)

*Corresponding Author

\section{INTRODUCTION}

Angiosarcoma is a rare vascular malignancy of endothelial differentiation. Treatment of angiosarcoma need multimodality approach with combined therapy of surgery, chemoand targeted therapy and radiation intervention. Still prognosis of angiosarcoma is grave as majority of patients present with metastatic disease. Management of terminal stage metastatic disease particularly that of the lung is important because pulmonary metastasis of angiosarcoma frequently presents with multiple symptoms and induces severe complications.

\section{CASE PRESEntation}

A 63-year-old hypertensive, non-diabetic, non-smoker Indian male presented with back pain radiating to thigh associated with cough and hemoptysis for 2-months duration. Magnetic resonance imaging (MRI) of lumbosacral spine showed multiple lesions in the lumbar vertebrae, sacral ala, and iliac bone. Whole body 18-fluorodeoxyglucose positron emission tomography-CT (FDG PET-CT) scan showed multiple areas of increased tracer uptake in bilateral humeri (SUV max 6.0), multiple cervico-dorso-lumbar vertebrae, bilateral scapulae, sternum (SUV max 6.5), multiple ribs bilaterally, sacrum (SUV max 7.3), bilateral ilia, bilateral acetabuli, bilateral ischia, trochanteric regions \& shaft of left femur and head of right femur (Fig. 1). Few rib lesions also showed involved soft tissue components. Both the lungs showed few FDG avid sub-centimetric parenchymal and pleural nodules.

Bronchoscopy showed streaky hemoptysis in left upper lobe apico-posterior segment. He underwent diagnostic right thoracoscopy with pleural and lung nodule excisional biopsy. Intraoperative findings revealed pleural deposit of $2.0 \times 1.0$ $\mathrm{cm}$ size in right anterior chest wall at 6 th intercostal space and sub-centimetric hard nodule in right upper lobe at the anterior fissure region. Post-operative period was uneventful. 


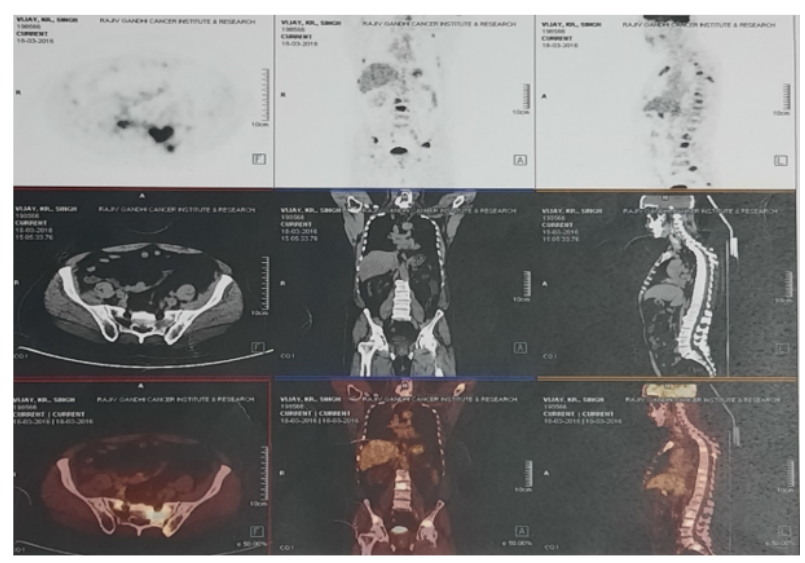

Fig. 1. An initial 18-FDG PET-CT scan showing increased metabolic activity in multiple bones and both lungs.

On histopathological examinations, right chest wall subpleural deposit showed tumor composed of spindle shaped epithelioid cells showing a peri-theliomatous arrangement. The individual cells were elongated having moderate to abundant pale eosinophilic cytoplasm with high grade nuclear atypia. Biopsy from lung nodules also revealed tumor cells composed of spindle shaped epithelioid cells with high grade neoplasia (Fig. 2). On Immunohistochemistry (IHC) staining, the tumor cells expressed widespread positivity for D2-40, cluster differentiation 31 (CD 31) \& CD34 and negative staining for cytokeratin (CK), smooth muscle actin (SMA), calretinin, S100, thyroid transcription factor -1 (TTF-1) \& p40. Histopathology and IHC profile favoured the diagnosis of angiosarcoma.
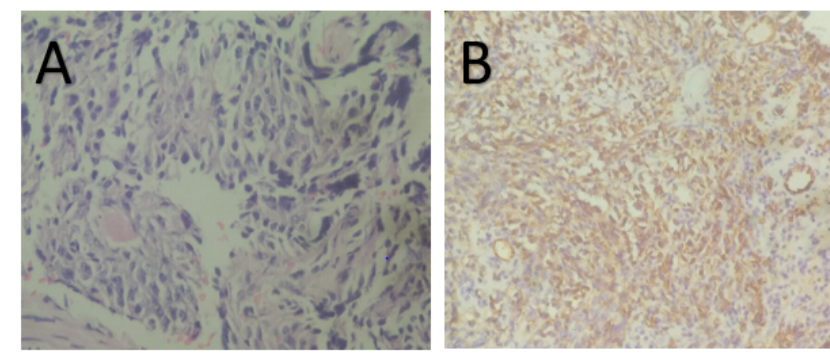

Fig. 2. Histopathological examination of the biopsy from pleural and lung nodules:

(A) In hematoxylin and eosin (H\&E) stain, tumor cells showing anastomosing spaces lined by endothelial cells.

(B) immunohistochemical stains of CD31 revealed positive expression.

Patient was planned for intravenous chemotherapy with paclitaxel $\left(80 \mathrm{mg} / \mathrm{m}^{2}\right.$ on day 1 , day $8 \&$ day 15 in a 28 -day cycle) along with zoledronic acid (4 mg four weekly). After 3-cycles of chemotherapy re-evaluation was done with FDG PET-CT scan. This scan was compared with initial one. Most of the bony lesions showed decrease in metabolic activity. Bilateral lung nodules and right pleural thickening were not seen as before implying reduction in size and activity. In view of stable disease, it was planned to extend the same chemotherapy regimen for three more cycles. However, after receiving two more cycles of chemotherapy, patient's condition deteriorated rapidly. Patient complained of rapid onset shortness of breath, weakness in both lower limbs \& decrease in appetite and within a week patient became paraplegic. Again, re-evaluation done with FDG PET-CT which revealed significant increase in number, extent and metabolically active progressive bony lesions. Unfortunately, patient died within a month.

\section{DISCUSSION}

Angiosarcoma, defined as endothelial cell tumor of vascular or lymphatic tissue, is a rare malignancy and constitutes for nearly 1 to $2 \%$ of all soft tissue sarcomas [1]. In most of the cases, the primary sites are the skin and the subcutaneous tissue of the head and neck region [2]. The predominant metastatic sites of angiosarcoma are lung including pleura, followed by bone and liver [3]. In a few cases, the disease develops in the context of lymphedema known as Stewart-Treves syndrome; and in some after prior radiation (particularly chest radiation for breast carcinoma) [4]. Early diagnosis of metastasis to pulmonary and bony region in angiosarcoma is hard, because of its rarity and the low index of suspicion. However, in the few available studies, the median age of metastatic angiosarcoma patients remains around 54 years (range 17-92 years) and males are more often affected than female [5]. Majority of the patients were metastatic at the time of initial presentation (44\%), and this incidence is twice that reported in other bone sarcomas [6]. The main prognostic factors were the stage of disease, age of patient and obviously the site of both primary and distant lesions. Patients usually present with symptoms depending upon the site of involvement; that include but not limited to hemoptysis, cough, dyspnea, chest pain, multiple bony pain and visibly weight loss. Imaging with PET can be useful in the diagnosis, staging, and follow-up of patients with angiosarcoma [7], [8]. In the case presented here, the FDG PET-CT scan confirmed its usefulness in identifying the regions and systems involved. Definitive diagnosis is made on the basis of histopathological and immunohistochemical findings. However, the histological features vary within and among cases. Abnormal, pleomorphic, malignant endothelial cells constitute the hallmark of angiosarcoma. These cells can be rounded, polygonal or fusiform with or without an epithelioid appearance. Diagnosis become more complicated in poorly differentiated areas, where the malignant endothelial cells form continuous monolayers, without any resemblance to parental endothelial cell except the epithelioid morphology. An angiosarcoma typically expresses endothelial markers including factor VIII-related antigen, CD34, CD31, and FLI-1. Among these markers, factor VIIIrelated antigen is the most specific but the least sensitive. Positivity for CD31 is relatively specific and extremely sensitive, being detected in approximately $90 \%$ of the cases [9]. Regarding treatment protocol, no standardized regimen has been established due to its rarity in occurrence. Patients with metastatic angiosarcoma have been treated with radiotherapy, surgical resection, immunotherapy, chemotherapy, or a combination of these [8], [10]. In metastatic patients, it is debatable to adopt aggressive surgical procedures against high morbidity since the low, if any, possibilities of achieving a surgical remission. Chemotherapy does not offer a better survival rate, but it is relevant to note that a significantly better 1-year overall survival was observed in the few patients who had a disease stabilization after chemotherapy. Overall, the goal of treatment in last stage metastatic angiosarcoma is palliation of symptoms and increase quality of life. Radiotherapy was mainly used with 
this palliative intent in metastatic patients and in localized patients when surgery was not feasible [11]. Chemotherapy with gemcitabine and docetaxel has been reported to produce a complete radiographic response [8]. In the present case, bilateral lung nodules diminished after three courses of chemotherapy with paclitaxel.

\section{CONCLUSION}

Pulmonary and multiple bony metastatic angiosarcoma is very rare disease with a very poor prognosis and no definite treatment so far. More cases have been reported in recent years with different therapeutic modalities, none of which proved to be effective.

\section{ACKNOWLEDGMENT}

None.

\section{REFERENCES}

[1] R. J. Young, N. J. Brown, M. W. Reed, D. Hughes, P. J. Woll. Angiosarcoma. Lancet Oncol. 2010;11(10):983-91.

[2] N. Penel, S. Marréaud, Y. M. Robin, P. Hohenberger. Angiosarcoma: state of the art and perspectives. Crit Rev Oncol Hematol. 2011;80(2):257-63
[3] N. Naka, M. Ohsawa, Y. Tomita, H. Kanno, A. Uchida, K. Aozasa. Angiosarcoma in Japan. A review of 99 cases. Cancer. 1995;75:989996.

[4] F. W. Stewart, N. Treves. Lymphangiosarcoma in postmastectomy lymphedema; a report of six cases in elephantiasis chirurgica. Cancer 1948;1(1):64-81.

[5] I. Shimabukuro, K. Yatera, S. Noguchi, Y. Kawanami, T. Iwanami, C. Nishida et al. Primary pulmonary angiosarcoma presenting with hemoptysis and ground-glass opacity: A case report and literature review. Tohoku J Exp Med. 2015;237(4):273-8.

[6] P. G. Casali et al. Bone sarcomas: ESMO-PaedCan-EURACAN Clinical Practice Guidelines for diagnosis, treatment and follow-up. Ann. Oncol. 29, iv79-iv95 (2018).

[7] G. Treglia, G. Cardillo, P. Graziano. A rare case of primary pulmonary epithelioid angiosarcoma detected by (18)F-FDG PET/CT. Clin Nucl Med. 2014;39(5):450-2.

[8] R. Wilson, S. Glaros, R. K. Brown, C. Michael, D. Reisman. Complete radiographic response of primary pulmonary angiosarcomas following gemcitabine and taxotere. Lung Cancer. 2008;61(1):131-6.

[9] W.D. Travis, E. Brambilla, H. K. Muller-Hermelink, C. C. Harris, editors. World Health Organization Classification of Tumours Pathology and Genetics of Tumours of the Lung, Pleura, Thymus and Heart. IARC Press: Lyon; 2004

[10] K. Kojima, I. Okamoto, S. Ushijima, T. Yoshinaga, M. Kitaoka, M. Suga et al. Successful treatment of primary pulmonary angiosarcoma. Chest. 2003;124(6):2397-2400.

[11] E. Palmerini, A. Leithner, R. Windhager, G. Gosheger, K. Boye, M. Laitinen et al. Angiosarcoma of bone: a retrospective study of the European Musculoskeletal Oncology Society (EMSOS). Sci Rep 10 10853 (2020). https://doi.org/10.1038/s41598-020-66579-5 